\title{
Hybrid Algorithm using the Advantage of Krill Herd Algorithm with Opposition- Based Learning for Dynamic Resource Allocation in Cloud Environment
}

\author{
P Neelima, A. Rama Mohan Reddy
}

\begin{abstract}
The cloud computing systems have more consideration due to the growing control for elevated concert computing and data storage. Resource allocation plays a vital role in cloud systems. To overcome the obscurity present in resources allocation system. In this paper, we design and develop a technique for dynamic resource allocation. A Hybridized approach is designed with the help of multi-objective oppositional krill herd optimization algorithm (OKHA). It is a combination of the krill herd algorithm and Opposition-based learning $(O B L)$ $O B L$ is added to get enhanced performance of the krill herd algorithm. The objective of this hybridization is to reduce the cost. In this Hybridized process each task consists of two cost i.e monetary cost and computational cost. Here each task is divided into many subtasks and assigns the respective resources to it. Our proposed multi-objective optimization algorithm will decide allocation of resource for the each subtask in this process. Finally, the testing is passed out, we evaluate our proposed algorithm with $P S O$, and $G A$ algorithm we verified the performance levels of our proposed Multi-objective optimization algorithm.
\end{abstract}

Keywords-Cloud computing, multi-objective, resources, task, krill herd algorithm, Opposition-based learning

\section{INTRODUCTION}

Many service crossways the internet using Cloud Computing technology, which is virtualized and become a milestone in world of networks. Cloud computing mostly targets fault-tolerant, elevated performance, consumption, scalability, high accessibility and easy to use, deal with, provision competence and cost-effective [1]. Since cloud computing has large-scale and complex architecture even though it makes the computing dependable, vibrant and easy, it still faces abundant challenges [2]. The researches on issues apprehend the adoption of cloud computing, being a comparatively recent phenomenon, by both organizations and persons who are in an emerging stage. On the adoption of CC technologies researches conducted so far, quite a small number have approached the problem from the perspective of associations and not from personal user's perspective [3].Resource allocation is plays a key role in cloud for providing customer needs[4].

From time to time the cost of different resources may vary. Resource allocation deals with providing resources within

Revised Manuscript Received on August 14, 2019.

P Neelima, Dept of CSE, Research Scholar, JNTUA, Andhra Pradesh, India.(Email: neelima.pannem@gmail.com)

Dr. A. Rama Mohan Reddy, Professor, Dept of CSE, SVUniversity,Tirupati, Andhra Pradesh, India.(Email ramamohansvu@yahoo.com) deadline for appropriate tasks allocating with proper utilization [6]. In the cloud resource optimization process many challenges has seen into consideration to get the needs of customer in allocation process[7].To handle resource allocation process, Resource Management System (RMS) is used in resource allocation process, the RMS acquire data about the used and unused resources existing in the system for proper utilization of resources to get profit. Next from the database of the RMS each computing resource will obtain a datum of subtask, and will work until the selected subtasks are completed [8]. A general solution is to shift Virtual Machines (VMs) far from stuffed servers to under-utilized one. To handle the enlarged requests, the process of relocation should consider all potential of operating current available resource, which may cause expenditure of resources might gradually become closely unbalanced between unusual resource dimensions are CPU, disk I/O, memory, bandwidth etc. that causes needless unused of accessible resources and acquire increased cost [9].

Ratan Mishra had given a another view on resource allocation based on the resources like CPU load, memory facility, delay or network load and other resources to customers of load complementary in cloud computing. Soumya Banerjee calculated the objective by examining all the requests in cloud environment to reduce the throughput in scheduling. By customized ant colony framework for expanded service allocation and scheduling mechanism [10]. Green SLA which was designed by Lizhe Wang by considering task execution time and energy-performance. To provide advanced options in power management an approach Dynamic Voltage and Frequency Scaling is proposed by Green SLA that holds up parallel execution and executes number of strategies [11]. A Hybrid algorithm is designed by merge the improvement of Ant Colony Optimization and Cuckoo Search to resolve the job scheduling troubles. Job Scheduling can handle systematic computing and elevated power computing for responding all the combinatorial optimizations problems [12]. The propagation performance of cuckoos and the uniqueness of Levy flights of flies initiate the new behavior, and then trail its success. By investigating other trendy optimization algorithms we will estimate the planned investigate plan and converse our findings and their presumption for a diversity of optimization tribulations [13]

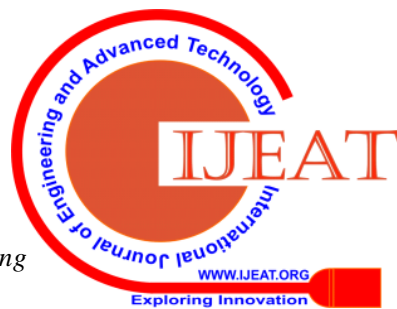




\section{HYBRID ALGORITHM USING THE ADVANTAGE OF KRILL HERD ALGORITHM WITH OPPOSITION- BASED LEARNING FOR DYNAMIC RESOURCE ALLOCATION IN CLOUD ENVIRONMENT}

A multi- objective optimization algorithm is designed for resource allocation in cloud computing will be done in dynamically resources need to be allocated based on the principle of tasks sub division. By consider the monetary cost and computational cost we design the multi-objective fitness. Here, an hybridized is applied between the krill herd algorithm with oppositional-based learning (OBL), by optimizing the cost function here an improvement is seen compare to previous findings. Firstly the OKHA algorithm is will generate the initial solution based on the number of subtask; secondly it calculates the multi-objective fitness for each krill of the initial solution. After that, we generate the opposite solution of the each task. Then, KHA algorithm updates the initial solution. The remaining paper is ordered as follows: in Section 2 brief reviews of several literature works in resource allocation. In Section 3 the proposed algorithm for dynamic resource allocation is discussed. Section 4 enclosed with proposed algorithm. Section 5 will presents the performance of different algorithms with tabbed values and section 6 will be conclusion of this paper.

\section{RELATED WORK}

Fatma A.Omara et al. [14] have developed an approach for resource allocation problem, which mainly focused two main modules, (1) Virtual Design Advisor (VDA) and (2) calibration process. In the first module, the VDA was used to estimate the cost of performance in the database management system (DBMS). To enhance and minimizing the cost function a Greedy Particle Swarm Optimization (GPSO) search algorithm was developed .

Siva Theja Maguluri [15] have elucidate important traffic resource allocation in cloud computing, which mainly used to study stochastic model of cloud computing . To overcome the problems in load balancing and scheduling a hybridized process is evolved with the combination of join-the- shortestqueue routing and power-of-two-choices routing algorithm. The main motivation is purely implemented on basis of queue length and it resembles in optimality in the heavy traffic limit. Rather than, S. Chandrasekhar and Paxar Rajnikant B [20] have explained about dynamic resource allocation in cloud computing based on priority.

There were presented many resources to satisfy costumer need in the dynamic resource allocation. These recourses were finished by service level agreement (SLA) and with the aid of parallel processing.

Either-or, Sagar S. Brahmkshatriya and Jignesh Prajapati [23] have explained and provided evaluation on techniques of resource allocation in cloud computing, which mainly focused in to two modules (1) virtualization and (2) automation. Initial module is facilitating the process of virtualization to maximize the profit for cloud providers and second module, was used to diminish the cost for cloud consumers. Moreover, Saraswathi AT et al. [18] have explained dynamic resource allocation in cloud computing, virtual machine (VM) technology is mainly concentrated. The main principle of Virtual machine works as with low priority jobs as well as with high priority jobs. Rather than, Dr.R.Sridaran et al. [19] have explained survey on resource allocation in cloud computing, which mainly used by Resource allocation strategy (RAS). RAS is developed to dynamically. The main motive of this algorithm is that,

carry out the objective of minimizing the cost in cloud by using the optimum resource algorithm. An algorithm was proposed for finding the cost in cloud. Moreover, Pavithra.B and Ranjana.R [21] have provided a detailed view on resource allocation in cloud computing by conducting a survey on virtual machines. The main objective of VM was to reduce the power consumption in large cloud data centers and with more profit.

To maximize the profit for cloud service provider B.Rajasekar and S.K.Manigandan [22] have described a powerful resource allocation in cloud computing. There is a problem is a raised by taking the resources as rental. so many limitations have seen while the users are considering the concept of Quality with his rented resources. Either or Fredy Juarez et al. [17] have explained Dynamic energy for Task based application in cloud computing based on parrellsim , To minimize the energy consumption a task based applications is executed in distributed computing platforms. This mainly focused virtualization technology (VT). VT was used for resource allocation by considering low level nodes addition as well as removal of nodes under allocating process.

\section{PROPOSED DYNAMIC RESOURCE ALLOCATION USING KRILL HERD ALGORITHM}

The main intention of this paper is develop a technique for dynamic resource allocation in distributed computing using Krill Herd Algorithm (KHA). Both monetary cost and computational cost is taken into consideration for scheduling tasks in cloud. A task is divided into various tasks and provides the selected best one as a available resource to the task to reduce the total cost of the task. The proposed KHA is used to allocate the task in to equivalent cloud resources by taking both monetary and computational tasks as input. Moreover, this KHA algorithm helps to decrease the cost of every subtask.

\subsection{Multi-objective function}

To achieve the optimal resource allocation, a multi objective function is developed. The multi objective function is defined as the combination of monetary cost and computational cost.

\section{Monetary cost:}

The cost that is allocated by the service provider to the client, to fulfill client's task and to develop the resources. The monetary cost differs from one provider to another for the execution of same task.

\section{Computational cost:}

It is decided by service provider depended upon the complexity of the task assigned by the user. This cost is varies at different times and computational complexities contain the execution time, memory space.

The proposed multi objective based resource allocation using oppositional krill herd algorithm step by step process is explained below.

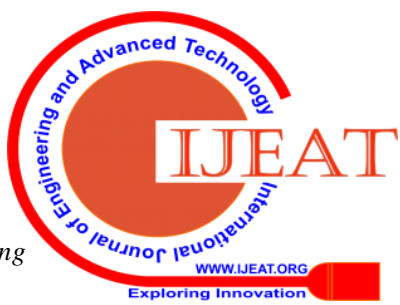




\section{Step 1: Solution encoding}

In cloud computing, an one of the important theme is to indicate a solution for resource allocation. At first we randomly generate the $Q_{i}$ number of solution. The solution consists of subtask and resources and the solution in the form of matrix. The solution matrix picks the resource for every subtask and the matrixes are efficient through KHA algorithm up to $K$ number of iteration after the calculation of fitness. Generate $Q$ number of initialization matrix with size of $\square N \square M \square$ where $N$ represents dimension of the matrix (number of resources) and $M$ represents the amount of cuckoo particles (number of subtasks). The initial solution format is given in table 1 and updation matrix is given in table 2 .

$\mathrm{Y}_{1}=$\begin{tabular}{|l|l|l|l|l|l|l|l|}
\hline $\mathrm{T}_{\mathrm{i}}$ & $R S_{1}$ & $R S_{2}$ & $R S_{3}$ & $R S_{4}$ & $R S_{5}$ & $R S_{6}$ & $R S_{7}$ \\
\hline$T_{S_{1}}$ & 0 & 0 & 0 & 1 & 0 & 0 & 0 \\
\hline$T_{S_{2}}$ & 1 & 0 & 0 & 0 & 0 & 0 & 0 \\
\hline$T_{S_{3}}$ & 0 & 0 & 0 & 0 & 1 & 0 & 0 \\
\hline$T_{S_{4}}$ & 1 & 0 & 0 & 0 & 0 & 0 & 0 \\
\hline$T_{S_{5}}$ & 0 & 1 & 0 & 0 & 0 & 0 & 0 \\
\hline
\end{tabular}

\section{Table 1: Example of initial solution}

\section{Step 2: Oppositional solution}

According to iTizhoosh in 2005 [24] opposition based learning (OBL) is implemented as, to get the updated krill position we are considering both the current and opposite krill position. The opposite krill solution has a better opportunity to get the details and update the current krill position based on global optimal solution. The opposite

krill's positions $\square O Y_{i} \square$ are completely defined by components of $Y_{i}$.

$$
\begin{aligned}
& O Y \square\left[o y^{1}, \ldots, o y^{d}\right] \\
& i \quad i \quad i \\
& \text { Where, } y^{d}=L^{d}+U^{d}-y^{d} \text { with } 0 y^{d} \in\left[L^{d}, U^{d}\right] \text { is the position of } i^{\text {th }} \text { opposite agent } O Y \text { in the with }
\end{aligned}
$$

dimension of opposite population.

\section{Step 3: Fitness calculation}

After that we calculate the fitness function of each solution. To calculate fitness function a multi-objective function is designed. In each task $T$ we calculate the fitness function.

The fitness of the task $T$ is intended through KHA notion. The total cost of the task $T$ is derived from the computational cost (objective 1; $F \square O_{1} \square$ ) and monetary cost (objective 2; $F$ $\square \mathrm{O}_{2} \square$ ) of the number of all subtasks $T_{s_{m}}$. Here, at first we calculate the computation cost $F \square O_{1} \square$ and further calculate the monetary cost $F \square O_{2} \square$. By the summation of computational cost of the all subtask by means of corresponding resources, the objective 1 is interconnected to the computational cost of the task $T$ that is calculated. The objective 2 is interrelated with the monetary cost, and calculated by the summation of computational cost of the every subtask with respective resources. To estimate the objective function 1 and 2 of the task $T$ and to calculate it, we use the following equations 1 and 2 correspondingly.

$F\left(O_{1}(T)\right)=\sum_{m=1}^{M} C C_{m}$
$M$
$F\left(O_{2}(T)\right)=\sum_{m=1} M C_{m}$
$F(T)=F\left(O_{1}(T)\right)+F\left(O_{2}(T)\right)$

\section{Step 4: Krill Herd Algorithm based solution updation}

Krill Herd Algorithm (KHA) is new optimization Algorithm that is developed for resolving optimization tribulations from optimization process. The main motivation for developing this krill optimization algorithm is, made-up to be an arrangement of the smallest amount distances of the location of the food and the maximum density of the herd. The KHA track the exploration orders that improve the objective function value and duplicate the implementation of the three movements. The $\mathrm{KH}$ algorithm is mainly follows the Lagrangian model for effective search which is shown in below equation (5);

$$
\frac{d v_{p}}{d t}=X^{n e w}{ }_{p}+X_{F^{v}}^{n e w}+X_{D_{p}}^{n s w}
$$

Where $X^{\text {new }}{ }_{p}$, is the motion bring by other krill individuals, $X^{\text {new }}{ }_{F}$, is the foraging motion and $X_{D_{p}}^{\text {new }}$ physical distribution of the krill individuals correspondingly.

\section{* Calculation of induction motion}

The movement of the krill individual $X^{n e w}$ is shown in equation (5):

$$
\begin{gathered}
X_{p}^{\text {new }}=\varphi X^{\max }+v X^{\text {old }} \\
\varphi_{p}=\varphi_{p}^{\text {local }}+\varphi_{p}^{t \arg e t}
\end{gathered}
$$

$X_{P}^{\max }$ is the maximum induced speed, $v_{W}$ is the

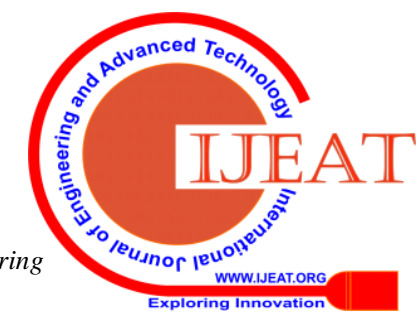




\section{HYBRID ALGORITHM USING THE ADVANTAGE OF KRILL HERD ALGORITHM WITH OPPOSITION- BASED LEARNING FOR DYNAMIC RESOURCE ALLOCATION IN CLOUD ENVIRONMENT}

inertia weight, $x_{P}^{\text {old }}$ is the motion, $\square$ local is the local effect obtainable by neighbors and $\varphi_{p}^{t} \underset{p}{\arg e t}$

is the best krill individual's target effect.

\section{* Calculation of Foraging Action}

The second progress of $\mathrm{KH}$ algorithm is Foraging action. It is derivative from two key factors. Firstly the current food location and earlier food location. The foraging velocity might be articulated for the $\mathrm{p}^{\text {th }}$ krill individual by

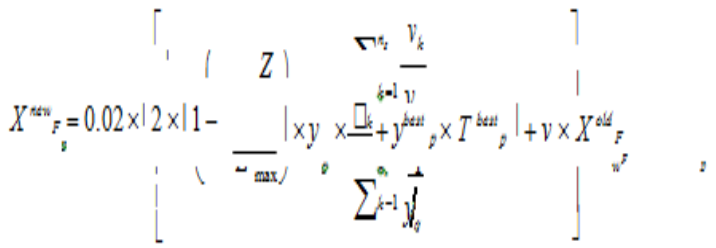

where ${ }^{\mathcal{V}} w_{F}$ is the inertia weight of the foraging motion, $X^{\text {new }} F$ and $X{ }^{\text {old }} F$ are the foraging motions of the new and the old $\mathrm{p}^{\text {th }}$ krill, correspondingly.

* Calculation of Physical diffusion

In this $\mathrm{KH}$ approach a physical diffusion is introduced.

$$
X_{D p}^{n e w}=\propto \times X_{D}^{\max }
$$

\section{* Position updation:}

In this procedure, derived from induction motion, foraging motion and physical diffusion motion, the individual Krill modifies its present positions and shifts to improved positions. The updated position of the $\mathrm{p}^{\text {th }}$ krill individual may be expressed as;

$$
v_{p}^{n r e^{\prime}}=v_{p}^{n e w}+\left(X_{p}^{n e w}+X_{F_{p}}^{n e w}+X_{D_{p}}^{n e w}\right) \times P c \sum_{q=1}^{n_{d}}\left(V_{q}-L_{q}\right)
$$

where $n$ is the total number of variables, $\mathrm{Vq}$ and $\mathrm{Lq}$ are the upper and the lower limits of the $\mathrm{q}^{\text {th }}$ variables.

After the updation of each dimension of every data point of the solution matrix, this named as updation matrix of the KHA algorithm which is represented in the following table 2.

\begin{tabular}{|c|c|c|c|c|c|c|c|}
\hline & $R_{1}$ & $R_{2}$ & $R_{3}$ & $R_{4}$ & $R_{5}$ & $R_{6}$ & $R_{7}$ \\
\hline$T s 1$ & 1 & 0 & 0 & 0 & 0 & 0 & 0 \\
\hline$T s 2$ & 0 & 1 & 0 & 0 & 0 & 0 & 0 \\
\hline$T s 3$ & 0 & 0 & 0 & 1 & 0 & 0 & 0 \\
\hline$T s 4$ & 0 & 1 & 0 & 0 & 0 & 0 & 0 \\
\hline$T s 5$ & 0 & 0 & 0 & 1 & 0 & 0 & 0 \\
\hline
\end{tabular}

Table 2: Updation table of KHA algorithm

After the conversion of the initialization matrix in to updation matrix, the OKHA algorithm starts its process by consider those updation matrixes as input.

\section{Step 5: Termination criteria}

If maximum value number of iterations is achieved then the best solution is find. After finding the best solution the algorithm will discontinues.. Once the best fitness is achieve by means of KHA algorithm, selected solution is allocated for task. Pseudo code of proposed methodology is given below:

\section{PROPOSED ALGORITHM}

Input: $M$ number of subtasks, $N$ Number of resources, monetary cost $M^{C}$, computational cost

$$
C^{C T}
$$

Output: Minimum cost of resource allocation for each subtask.

Initialize the Parameters:

$N=$ Number of resources

$M=$ Number of subtasks

$M^{C}=$ Monetary cost

${ }_{C}{ }^{C}=$ Computational cost

$k=$ Iteration counter

$K=$ maximum iteration counter

$f \square t^{k} \square=$ fitness of $\mathrm{m}^{\text {th }}$ subtask at $\mathrm{k}^{\text {th }}$ iteration

${ }_{C}{ }^{C} m n=$ communication cost of $\mathrm{m}^{\text {th }}$ task

with $\mathrm{n}^{\text {th }}$ resource

$M^{C} m n=$ monetary cost of $\mathrm{m}^{\text {th }}$ task with $\mathrm{n}^{\text {th }}$ resource

Step 1: Start

Step2: Randomly generate the krill individual Evaluate the fitness $\left(F F_{i}\right)$ function and find out best and worst solution

Set cycle to 1

Step 3: While (stopping condition is not met ) do Store the pre-specified number of best krill For each krill Calculate the movement influenced by other krill individual 
using (5) Calculate foraging action using (7)

Step 4: Calculate physical diffusion using(8) update krill position using (9)evaluate the objective function value and update the krill individual end for replace the worst krill with the best krill end while

Step 5: Best solution

\section{PERFORMANCE EVALUATION OF PROPOSED ALGORITHM \& RESULTS}

The primary purpose of this research is to design and develop a method for dynamic resource allocation through the help of multi-objective optimization algorithm. Our main objective is to allocate the resource with minimum computational costs. Here, the performance evaluation of the proposed multi objective optimization algorithm is prepared by compared with the particle swarm optimization and particle swarm optimization genetic algorithm algorithm are shown in table 3. The parameters used in the krill herd algorithm are shown in table 4

\begin{tabular}{|c|c|c|c|c|c|c|}
\hline \multirow{2}{*}{$\begin{array}{l}\text { Number of } \\
\text { resources }\end{array}$} & \multicolumn{3}{|c|}{ D1 dataset } & \multicolumn{3}{c|}{ D2 dataset } \\
\cline { 2 - 7 } & Proposed & PS0 & GA & Proposed & PS0 & GA \\
\hline 25 & 22.50 & 27.99 & 27.63 & 20.52 & 25.10 & 25.4 \\
\hline 50 & 22.0 & 27.5 & 27.00 & 21.19 & 24.21 & 25.11 \\
\hline
\end{tabular}

Table 3: Represents the average performance of the three algorithms

\begin{tabular}{|c|c|c|c|c|c|c|c|}
\hline Pop_size & $\operatorname{maxGen}$ & $\omega_{\mathrm{n}}$ & $X_{P}^{\max }$ & $€$ & rand & $v_{w_{r}}$ & $X_{\nu}^{\text {nev }}$ \\
\hline $\begin{array}{c}\text { 2D } \\
\text { (Dimension) }\end{array}$ & 100 & 0.1 & $\begin{array}{c}0.01 \mathrm{~ms}^{-} \\
1\end{array}$ & 0.1 & 0.1 & $\begin{array}{c}0.02 \mathrm{~ms}^{-} \\
1\end{array}$ & $\begin{array}{c}0.002 \mathrm{~ms}^{-} \\
1\end{array}$ \\
\hline
\end{tabular}

.Table 4: Parameter used in the KHA

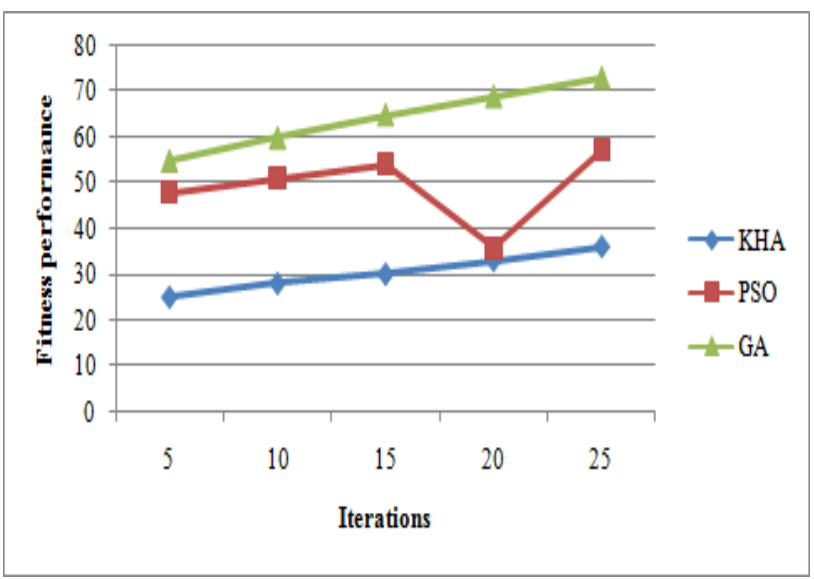

Figure 1: Performance of fitness function for KHA

\section{CONCLUSION}

In this paper, an approach of multi-objective optimization algorithm is designed for dynamic resource allocation in cloud computing. Firstly, the OKHA algorithm will produce a solution matrix based on the amount of subtasks and then it computed the fitness for each and every subtask based on the evaluation of costs (monetary and computational costs). After that, the solution is updated based on the oppositional krill herd algorithm. Here, we hybridization takes place between the krill herd algorithm and oppositional -based learning (OBL), OBL is used to optimize the cost function which will resembles in improving the performance of the previous krill herd algorithm. The updated solution matrix is assigned to OKHA algorithm. The process will be iterated up to $\mathrm{K}$ times. The experimentation is conceded out, we compared our proposed algorithm with GA and PSO algorithm, and we proved efficiency of our proposed multiobjective optimization algorithm.

\section{REFERENCES}

1. Madni, Syed Hamid Hussain et al. "Resource Scheduling For Infrastructure As A Service (Iaas) In Cloud Computing: Challenges And Opportunities", Journal of Network and Computer Applications, Vol.68, 2016.

2. Sharma, Yogesh et al. "Reliability And Energy Efficiency In Cloud Computing Systems: Survey And Taxonomy", Journal of Network and Computer Applications, 2016.

3. Sharma, S. K., Al-Badi, A. H., Govindaluri, S. M. and Al-Kharusi, M. H. "Predicting motivators of Cloud Computing Adoption: A developing Country Perspective", Computers in Human Behavior, Vol.62, PP. 61-69, 2016.

4. Kong, Weiwei, Yang Lei, and Jing Ma. "Virtual Machine Resource Scheduling Algorithm for Cloud Computing based on Auction Mechanism", Optik - International Journal for Light and Electron Optics 127.12, 2016.

5. Hadi Goudarzi and Massoud Pedram, "Maximizing Profit in Cloud Computing System via Resource Allocation", $31^{\text {st }}$ International Conference on Distributed Computing Systems Workshops, 2011.

6. Tsai, Jinn-Tsong, Jia-Cen Fang, and Jyh-Horng Chou. "Optimized Task Scheduling And Resource Allocation On Cloud Computing Environment Using Improved Differential Evolution Algorithm",Computers \& Operations Research, Vol 40, No.12, 2013.

7. Sun, $\mathrm{Yu}$ et al, Jules White, Sean Eade, Douglas C. Schmidt, "ROAR: A Qos-Oriented Modeling Framework for Automated Cloud Resource Allocation And Optimization", Journal of Systems and Software, Vol.116, PP. 146-161, 2016.

8. Wei-ChangYeh,Shang ChiaWei, "Economic based Resource Allocation for Reliable Grid-Computing Service based on Grid Bank", Future Generation Computer System, Vol 28, PP.989-1002, 2012.

9. Hassan, Mohammad Mehedi and Atif Alamri. "Virtual Machine Resource Allocation for Multimedia Cloud: A Nash Bargaining Approach". Procedia Computer Science, Vol.34, PP. 571-576, 2014.

10. Mingyue Feng, Xiao Wang, Yongjin Zhang, Jianshi Li "Multi-Objective Particle Swarm Optimization for Resource Allocation In Cloud Computing", IEEE CCIS, PP.1161-1165, 2012.

11. Giuseppe Portaluri, Stefano Giordano, Dzmitry Kliazovich, Bernabe Dorronsoro, "A Power Efficient Genetic Algorithm for Resource Allocation in Cloud Computing Data Centers", IEEE 3rd International Conference on Cloud Networking,PP.58-63,2014.

12. Babukartik, R.G. "Hybrid Algorithm Using The Advantage Of ACO And Cuckoo Search For Job Scheduling". International Journal of Information Technology Convergence and Services 2.4 (2012): 25 34.

13. Yang XS, Deb S, "Cuckoo search via Lévy flights", proceedings of World Congress on Nature \& 
HYBRID ALGORITHM USING THE ADVANTAGE OF KRILL HERD ALGORITHM WITH OPPOSITIONBASED LEARNING FOR DYNAMIC RESOURCE ALLOCATION IN CLOUD ENVIRONMENT

Biologically Inspired Computing, India, pp 210-214, 2009.

14. Fatma A.Omara, Sherif M. Khattab, Radhya Sahal "Optimum resource allocation in cloud computing" Published on 2014.

15. Siva Theja Maguluri, R. Srikant, Lei Ying "Heavy traffic algorithm for resource allocation in cloud computing" published on 2014

16. JavierBajo, Fernando De La Pritea, Sara Rodríguez "Low level resource allocation for cloud computing" published on 2016

17. Fredy Juarez, Jorge Ejarque, Rosa M. Badia "Dynamic energy for parallel Task based application in cloud computing" published on 2016.

18. Saraswathi AT , Kalaashri.Y., Dr.S.Padmavathi " Dynamic energy resource allocation for cloud computing" published on 2015

19. Dr.R.Sridaran, Dr.PadmavathiGanapathi , V.Vinothina "Survey on resource allocation in cloud computing" published on 2015

20. Chandrashekhar S. Pawar, Pawar Rajnikant B "Priority based dynamic resource allocation in cloud computing" published on 2014

21. Pavithra.B, Ranjana.R.” survey on energy informed resource allocation in cloud computing" published on 2015

22. B.Rajasekar, S.K.Manigandan "powerful resource allocation In cloud computing" published on 2015

23. Sagar S. Brahmkshatriya, Vadodara "evaluation on techniques of resource allocation in cloud computing" published on 2015

24. H. R. Tizhoosh, "Opposition-based reinforcement learning", Journal of Advanced Computational Intelligence and Intelligent Informatics, vol.10, no. 3, 2006. 